\title{
A Difficult Pill to Swallow: An Investigation of the Factors Associated with Medication Swallowing Difficulties
}

This article was published in the following Dove Press journal: Patient Preference and Adherence

\author{
Chandramouli Radhakrishnan' \\ Aida Sefidani Forough $\left(\mathbb{D}^{2}\right.$ \\ Julie AY Cichero iD ${ }^{1,2}$ \\ Heather E Smyth (iD ${ }^{3}$ \\ Aisha Raidhan ${ }^{4}$ \\ Lisa M Nissen (iD) ${ }^{1,2}$ \\ Kathryn J Steadman (iD) ${ }^{1,2}$ \\ I School of Pharmacy, The University of \\ Queensland, Brisbane, Queensland, \\ Australia; ${ }^{2}$ School of Clinical Sciences, \\ Queensland University of Technology \\ (QUT), Brisbane, Queensland, Australia; \\ ${ }^{3}$ Queensland Alliance for Agriculture and \\ Food Innovation, The University of \\ Queensland, Centre for Nutrition and \\ Food Sciences, Brisbane, Queensland, \\ Australia; ${ }^{4}$ Department of Pharmacy, \\ King's College London, London, UK
}

Background: Many medications are available as solid oral dosage forms such as tablets and capsules; however, some people find these medications difficult to swallow.

Aim: To identify whether certain psychological, oral sensory, and oral motor characteristics contribute to medication swallowing difficulties.

Methods: A sample of healthy adults from two academic institutions in Brisbane were assessed for their experiences with swallowing solid oral dosage forms, food preferences, and food neophobia. The gag reflex, oral cavity size, fungiform papillae count, and chewing efficacy were also evaluated followed by a capsule-swallowing task. Primary outcome was the incidence of medication swallowing difficulties. Secondary outcomes were the association of medication swallowing difficulties with psychological, oral sensory, and oral motor factors.

Results: Of 152 subjects, $32 \%$ reported difficulty swallowing tablets or capsules whole. This group was significantly more likely to have had a memory of choking on medications compared to those without medication swallowing difficulties $(\mathrm{OR}=7.25, \mathrm{p}<0.05)$. Current medication swallowing difficulties were significantly associated with a smaller mouth cavity size $(\mathrm{OR}=2.98, \mathrm{p}<0.05)$, a higher density of taste receptors on the tongue $(\mathrm{OR}=3.27, \mathrm{p}<0.05)$, and were higher among those who chewed a jelly candy to nonhomogenous particle size $(\mathrm{OR}=4.1, \mathrm{p}<0.05)$. Current medication swallowing difficulties were associated with lower confidence in swallowing large capsules ( 000 size: $O R=0.47,00$ size: $\mathrm{OR}=0.39, \mathrm{p}<0.05)$. No associations were found between medication swallowing difficulties and the gag reflex or food neophobia.

Conclusion: A combination of heightened oral perception characterized by a small oral cavity and high taste sensitivity compounded by a past choking episode on medications may be precipitating factors for medication swallowing difficulties. These factors may be helpful in identifying individuals who are more likely to experience difficulty swallowing medications.

Keywords: dysphagia, medication swallowing, oral dosage forms, swallowing difficulties, tablets

\section{Introduction}

Solid oral dosage forms such as tablets and capsules are the cheapest, most common, and preferred route of medication administration compared to other dosage forms. ${ }^{1}$ However, some people find it difficult to swallow them whole. People diagnosed with dysphagia have a physiological problem with swallowing and find it difficult to safely swallow solid foods and/or liquids as well as oral medications. ${ }^{2,3}$ Although difficulty with swallowing medications, particularly solid
Correspondence: Kathryn J Steadman

Tel +6I 733461886

Fax +6I 733461999

Email k.steadman@uq.edu.au 
dosage forms, has been largely documented in people with dysphagia, ${ }^{4,5}$ the general community without dysphagia can also experience medication swallowing difficulties. In the limited range of surveys of the general adult population without dysphagia that have been conducted, the proportion reporting some degree of difficulty swallowing solid oral dosage forms is typically around $15-20 \%$, with numbers ranging from $6 \%$ through to $50 \%{ }^{6-11}$ Importantly, consumers are unlikely to seek advice from a health professional before splitting or crushing their solid dose form. ${ }^{11}$ In order to improve their medication management, we are interested in better identifying individuals who are more likely to be at risk of experiencing medication swallowing difficulties.

There are certain physiological differences between people that we hypothesize may be associated with a greater or reduced likelihood of experiencing medication swallowing difficulties. Solid oral dosage forms are designed to be swallowed whole. The ability to swallow a solid dose whole relies on the person's ability to over-ride their chewing reflex - as a conditioned response to the presence of oral content, as well as their gag reflex - the innate protective reflexes guarding against choking or airway invasion. ${ }^{12}$ What factors contribute to triggering these reflexes while swallowing a solid dose medication, and how might they be identified? Returning to first principles, a reflex arc is involuntary and relatively immediate. It is initiated by sensory receptors being excited, travels via sensory neurons to the spinal cord and results in a fairly immediate motor response. Oral sensory perception, therefore, is a critical feature to explore. The gag reflex is a contraction of the muscles of the back of the throat and the back of the tongue to protect the airway by providing a physical barrier to entry of foreign objects or food items that are too large to be swallowed. Activation of the gag reflex results in the object being pushed forwards, towards the front of the mouth. It may be triggered by touching the back of the tongue, tonsils, soft palate or back of the throat. How much stimulation is required to trigger the gag reflex varies from person to person, with some studies showing that the gag reflex reportedly does not exist in $37 \%$ of healthy people. ${ }^{13}$

When food items have been improperly chewed, the gag reflex may be triggered to bring the food back into the oral cavity proper for further chewing. ${ }^{14}$ Feedback from oral receptors is necessary to guide chewing strength, movement and duration. ${ }^{14}$ Insight into a person's natural chewing style and the size of the bolus (large/small) they naturally choose to swallow can be gained from observing the results of a chewing task. A number of studies have shown that food items, regardless of the initial bite-size, are chewed so that each particle is generally $<4 \mathrm{~mm}$. ${ }^{15,16}$ A person who is comfortable to swallow large particles may find it easier to swallow solid dose medications than a person whose sensory receptors require smaller particle sizes before swallowing.

The volume of the oral cavity can vary in size. The tongue provides the floor of the mouth, the buccinator muscles provide bulk to the cheeks laterally. The hard palate provides the roof of the mouth, and when looking at the back of the the mouth the soft palate can be seen with the uvula hanging down in the midline. People with a large tongue, a flat or low hard palate and excess tissue in the cheeks present with oropharyngeal crowding. The crowding of hard and soft tissues reduces the space within the oral cavity and provides a greater opportunity for food or solid doses to come in contact with sensory receptors, potentially triggering the chewing or gag reflexes more easily. The Mallampati scale is a classification system to predict difficult intubation and bag-mask ventilation based on the size of the oral cavity. ${ }^{17}$ A classification such as this can potentially be used in studying associations between the mouth cavity size and medication swallowing difficulties resulting from the heightened sensory perception of medications in the mouth.

Although the entire oral cavity is rich with sensory receptors, the tongue is especially sensitive. When tablets and capsules touch the surface of the tongue, the sensory systems, particularly the fungiform papillae containing the taste buds, are activated. ${ }^{18}$ It is suggested that the sensation of certain tastes may affect the swallowing function by delaying the pharyngeal phase. ${ }^{19}$ A higher density of fungiform papillae (which house the taste buds) or having "supertaster" status increases the sensory awareness of the material in the mouth and is reportedly associated with the rejection of certain types of foods. ${ }^{20}$ Taken together or in isolation, these markers of oral sensation may provide an indication of individuals who are likely to have difficulty swallowing solid dose medicine. Therefore, we will investigate whether people who experience medication swallowing difficulties have a strong gag reflex, greater oropharyngeal crowding, or higher sensory awareness and fungiform papillae density.

While physical stimulus can produce a reflexive response, a psychogenic stimulus can also cause a physical response. A previous experience or situation 
can affect a person's response with conditioned taste aversions being a protective mechanism in humans and animals to protect them from food containing toxins. ${ }^{21}$ Aversions can occur from as little as a single exposure. Therefore, psychological factors should also be recognized in medication swallowing difficulties. Increased sensory awareness in severe form can lead to food neophobia, ie fear of eating new or unfamiliar food. ${ }^{22}$ The acceptance of new products in people especially those with neophobia may often be influenced by texture, appearance, taste, smell, and color ${ }^{23,24}$ - similar factors that also influence the acceptability of oral medications. ${ }^{2,7,8}$ A memory of a past unpleasant experience such as tablets being stuck in the throat or episodes of choking on medication have been reported as reasons for a dislike and aversion to taking medications. ${ }^{8,25,26}$ Previous experience of esophageal damage and irritation by medications ${ }^{27}$ may also lead to subsequent fear of swallowing solid doses whole.

Other factors that may influence success in swallowing solid dose medications include body position while taking the medicine and the use of a liquid carrier. For instance, certain postures such as upright/neutral head position and leaning forward (for capsules) help with bolus transport, ${ }^{28-30}$ and at least $50-60 \mathrm{~mL}$ water is recommended to ensure efficient transit through the esophagus. $^{29,31}$ Consumption of less than $60 \mathrm{~mL}$ or swallowing the medication with saliva can lead to lengthy delays of the medication in the esophagus, providing the potential for esophageal injury.

This study investigated the extent of medication swallowing difficulties in a general adult non-dysphagic population, and considered associations with a range of potential physiological and psychological factors. In addition to participant self-report, participants were engaged in a capsule-swallowing activity so that we could observe their approach to medication swallowing in practice. It was hypothesized that factors that demonstrate increased oral sensitivity, and/or psychogenic stimuli related to previous experience with choking would predict individuals with difficulty swallowing solid dose medications.

\section{Materials and Methods}

This was a cross-sectional study of a convenience sample of adults working or studying within two research institutions in Brisbane, Australia who were invited to participate in the study. Participants were provided with an information sheet about the project, the process of the study, and their involvement, as well as the dates, times, and location of data collection. Healthy volunteers aged 18-65 years were included in the study. Participants completed all tests in a single session taking approximately 20 minutes per person during Nov 2011. This study was conducted in accordance with the Declaration of Helsinki. Ethical approval was obtained from the University of Queensland Medical Research Ethics Committee (Approval number 2011/111). The participants provided their written informed consent prior to the study.

Participants completed a questionnaire consisting of a combination of closed multi-response questions and openended questions to elicit their perception of difficulties with swallowing tablets or capsules, memory of choking incidents on food and medication and coping strategies used to make swallowing medications easier (Supporting Information).

After completing the questionnaire, the participants were provided with one capsule sample of each commonly available sizes between 000 and 4 packaged separately in a monitored dosage system (Webster-Pak). After visually inspecting the size of the capsules, the participants were asked to rate whether they felt confident about their ability to swallow each size.

To assess participant preferences towards different food textures, a 22-item food preference questionnaire was used. The questionnaire asked about willingness to try 22 different foods based on their texture and rating them on a 5-point Likert scale (5 indicating extreme like and 1 indicating extreme dislike). This was followed by a 10-item food neophobia questionnaire in True/False format asking questions regarding participant preferences and willingness to try new and unfamiliar foods. Classification of food neophobia was incurred if "True" was selected for six or more questions. ${ }^{22}$ All survey questions were initially piloted on a group of pharmacy practitioners and postgraduate students for clarity of content.

To examine the gag reflex, the subjects were asked to open their mouth in a comfortably seated position. Using a wooden spatula, the examiner (an experienced speech pathologist with more than 20 years of experience) firmly touched the right side of the tongue starting at the lateral midline, posterior tongue, and the faucial pillar. The gag reflex was defined as constriction of the pharynx in response to the stimulus. As soon as a response was observed on the right side of the mouth, the examiner moved to test the left side starting again from the lateral midline. If no response was observed from the sides of the tongue and the faucial pillars, the tongue depressor was 
gently pushed against the midline of the pharyngeal wall at the back of the throat.

The modified Mallampati classification is used in anaesthesia to predict the ease of endotracheal intubation, by examining the visibility of oropharyngeal structures when the subjects are in a seated position with their mouth fully open and tongue protruding. The higher Mallampati scores (Class III \& IV) indicate a smaller mouth cavity and are associated with difficult intubation. ${ }^{17}$ The mouth cavity size was classified by an experienced speech pathologist as Class I if the soft palate, fauces, uvula, and pillars were visible, Class II if the soft palate and fauces were visible but less of the uvula, Class III if the soft palate and only the top of uvula were visible, and Class IV if the soft palate was not visible at all.

The method for counting fungiform papillae density was similar to Shahbake et $\mathrm{al}^{32}$. Following the rinsing of the mouth with water, petroleum jelly (Vaseline) was applied to the lower lip to reduce the staining of extraoral tissues. Methylene blue $0.5 \%$ food coloring (Queen Fine Foods, Alderley, QLD) was applied to the tongue with a sterile cotton tip applicator. The participants were then asked to swallow once to remove the excess dye. A disposable plastic slide, pre-labelled with reference number, was adhered to the front part of the tongue so that the midline of the tongue bisected the $1 \mathrm{~cm}^{2}$ window. Both the fungiform and filiform papillae stain in light blue with methylene blue allowing the fungiform papillae that contain the taste buds to be distinguished from filiform papillae by their larger size and mushroom-like shape. Digital images of the tongue were taken using a Nikon D60 digital camera (Nikon Corp, Japan). Counts of fungiform papillae were used to classify participants into three groups of low or non-tasters $\left(<15 \mathrm{~cm}^{-2}\right)$, medium tasters $\left(15-24 \mathrm{~cm}^{-2}\right)$, and supertasters $\left(\geq 25 \mathrm{~cm}^{-2}\right)$.

To understand the effect of oral motor capabilities on medication swallowing difficulties, a task was devised to examine chewing efficiency and particle size reduction. The participants were provided with a piece of confectionary jelly snake that was $5 \mathrm{~cm}$ in length and were asked to "chew it as they usually chew their food". When they felt a readiness for swallowing they were asked to spit the jelly into a plastic bag provided. The jelly snake was chosen to minimize differences in texture or consistency such as those that can be observed in fresh products (eg fruit or vegetables). Moreover, the chewy texture required chewing effort, and the sample did not disintegrate with saliva or become stuck to the teeth while resulting in a bolus that could be assessed easily for particle size once expectorated. The reference particle size was chosen as $4 \mathrm{~mm}$ based on the previous study on healthy young people with good oral health. ${ }^{33}$ The size of the chewed particles was measured with a ruler and classified into five different categories: All particles are very small $(<4 \mathrm{~mm})$, most particles are very small $(<4 \mathrm{~mm})$, roughly equal numbers of small and large particles, most particles are large $(>4$ $\mathrm{mm})$, all particles are large $(>4 \mathrm{~mm})$.

Participants were observed while swallowing a size 00 clear gelatine capsule (PCCA, Matraville, NSW, Australia) filled with small round pellets of "100s and 1000s" confectionary (Dollar Sweets Company, VIC, Australia) to provide weight to the capsule. A disposable plastic cup $(200 \mathrm{~mL})$ and a jug of water were provided, but attention was not drawn to these items. The participants were then asked to "swallow the capsule as they normally would". The examiner (an experienced speech pathologist) noted whether the participant had completed the task and if so, whether it was completed in a single attempt or multiple attempts, the approximate quantity of water used (full, half, quarter, less than a quarter, none), whether the capsule was placed at the front, middle or back of the mouth, and if their head position deviated from neutral.

\section{Statistical Analysis}

Data were collated in the Microsoft Excel 2019 and were analysed using SPSS (V25.0 for Windows, Armonk, NY). Statistical associations were determined using Pearson's Chi-square test or Fisher's exact test where appropriate. The statistical significance was set at $p<0.05$. The STROBE cross-sectional reporting guidelines were used to report the findings of the study. ${ }^{34}$

\section{Results}

A total of 152 volunteers with an age range of 19 to 66 years old took part in the study. There were more female (64\%; age range $20-66$ years) than male (36\%; age range 19-62 years) participants. The median age was 36 years for both genders. Two-thirds (100/152) reported taking tablets or capsules on a regular basis (ie at least weekly).

Of the 152 participants, $32 \%$ (49/152) indicated that they currently have trouble swallowing tablets or capsules, and $55 \%(84 / 152)$ had experienced difficulties with swallowing medications at some time in their life. There was no significant relationship of medication swallowing difficulties with age or gender. One-third of the participants (49/151) reported that at some point they have cut or crushed 
a medication before swallowing, while almost half (71/151) reported that someone, such as a parent, had at some time crushed their medications to make it easier for them to swallow (Table 1). Only 19\% (28/151) thought that they had trouble learning to swallow tablets or capsules. Participants who reported medication swallowing difficulties were significantly more likely to have cut or crushed their medication $(26 / 49 ; 53 \%)$ compared to those without current medication swallowing problems $(23 / 103 ; 22 \%)(\mathrm{OR}=3.93$, $\mathrm{p}<0.05)$. Of the 23 participants who crushed tablets without reporting current medication swallowing difficulties, 14 indicated an experience of medication swallowing difficulties in the past.

Although 30\% (44/149) of participants reported that they can swallow the capsules/tablets without any vehicle, most participants reported using multiple approaches to swallow medications, with $97 \%$ (148/152) using a liquid such as water and $85 \%$ (126/148) using food, $42 \%(62 /$ 147) placing medication at the back of their mouth and $51 \%$ (76/149) tipping their head back (Table 1).

Of the 49 people with current medication swallowing difficulties, 28 had a recollection of choking on medications. Those with current medication swallowing difficulties were significantly more likely to have had a choking episode on medications than those without current medication swallowing difficulties ( $57 \%$ vs $16 \%$; OR $=7.25$; $\mathrm{p}<0.05$ ) (Figure 1A). One-quarter of the participants (38/ $149,26 \%$ ) reported having previous experience of choking on food. There was no correlation between current medication swallowing difficulties with having a memory of choking on food $(p=0.27)$ (Figure 1B). The food preference questionnaire indicated a significant correlation between current medication swallowing difficulties with a lower preference score for potato chips/crisps $(4.1 \pm 0.0$ vs $4.5 \pm 0.7)$, popcorn $(3.6 \pm 1.1$ vs $4.0 \pm 1.0)$ and granola bars $(3.6 \pm 1.1$ vs $4.0 \pm 0.39)(\mathrm{p}<0.05)$. All the low preference food items were hard, dry, and were particulate in texture. In fact, $46 \%$ (70/152) of the participants exhibited food neophobia, ie indicated a positive response to 6 or more out of the 10 questions, but this was not associated with current medication swallowing difficulties ( $p$ $=0.60$ ).

All the participants believed that the smallest capsule, size 4, appeared easy to swallow (Table 2). More than $90 \%$ of the participants were comfortable with swallowing hard gelatine capsules to size 1 . However, as the size increased participants became less confident of their ability to swallow the capsule. For the largest size (000), only 63\% (96/ 152) of the participants believed that they could swallow the capsule (Table 2). Those with current medication swallowing difficulties were significantly less likely to feel confident about swallowing capsule sizes of 000 and 00 compared to those without current medication swallowing difficulties $(000$ size: $\mathrm{OR}=0.47 ; 00$ size: $\mathrm{OR}=0.39$, respectively; $\mathrm{p}<0.05$ ).

No gag reflex was exhibited by $42 \%(n=64 / 152)$ of the participants, ie they did not respond to the pressure of the tongue depressor at any of the locations in the oral cavity. When the gag reflex was present, it was mostly triggered from the back dorsum (74/88) or faucial pillar (71/88). There was no correlation of age or gender with the presence of the gag reflex. Moreover, there was no significant relationship between the presence of the gag reflex with medication swallowing difficulties $(\mathrm{p}=0.73)$.

The size of the mouth cavity was classified into four groups using the modified Mallampati classification. For ease of between-group comparisons, Class I and II were regrouped as the "large mouth cavity" group and Class III and IV were combined as the "small mouth cavity" group. Participants were fairly evenly split between the large

Table I Response to Questions Related to Medication Modification and Swallowing

\begin{tabular}{|l|l|l|}
\hline Question & Yes, n (\%) & No, n (\%) \\
\hline $\begin{array}{l}\text { Have you ever cut or crushed a medication to make it easier to swallow? } \\
\text { Has anyone else (eg a parent), ever crushed a medication for you, to make it easier to swallow? }\end{array}$ & $\begin{array}{l}49(32.5) \\
71(47.0)\end{array}$ & $\begin{array}{l}102(67.5) \\
80(53.0) \\
123(81.5)\end{array}$ \\
\hline Did you have trouble learning to swallow tablets or capsules? & $28(18.5)$ & \\
\hline The following items or manoeuvres help me to swallow tablet/capsules: & $62(42.2)$ \\
- Placing the tablet/capsule at the very back of my mouth & $76(51.0)$ & $85(57.8)$ \\
- Tipping my head back as I swallow the tablet/capsule & $148(97.4)$ & $73(49.0)$ \\
- Swallowing the tablet/capsule with a liquid (eg water) & $126(85.1)$ & $4(2.6)$ \\
- Swallowing the tablet/capsule with food & $44(29.5)$ & $22(14.9)$ \\
- Swallowing the tablet/capsule by itself & $105(70.5)$ \\
\hline
\end{tabular}




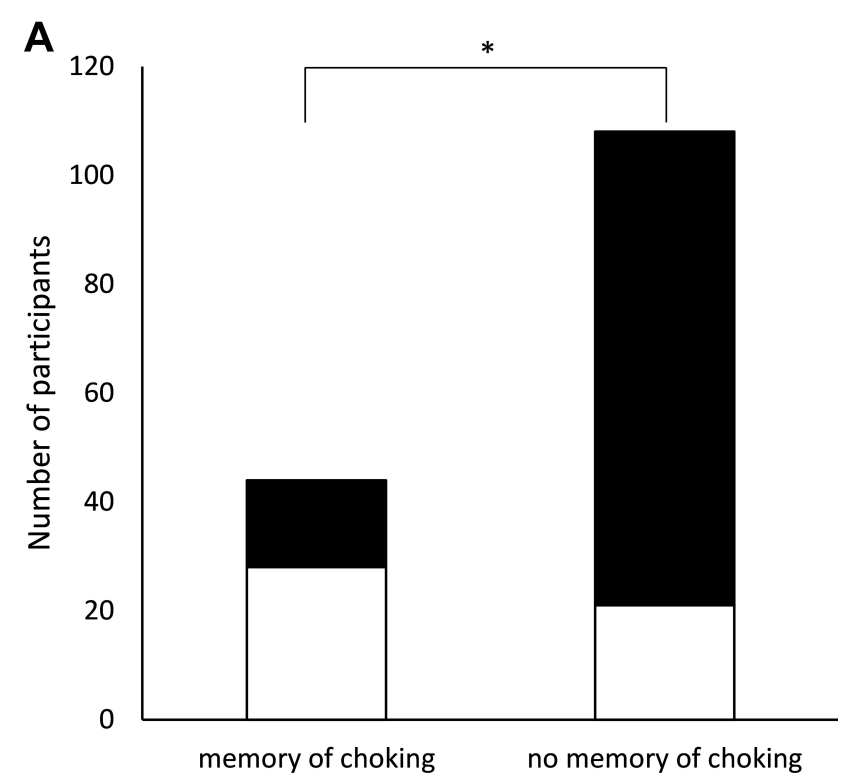

no medication swallowing difficulties

$\square$ medication swallowing difficulties

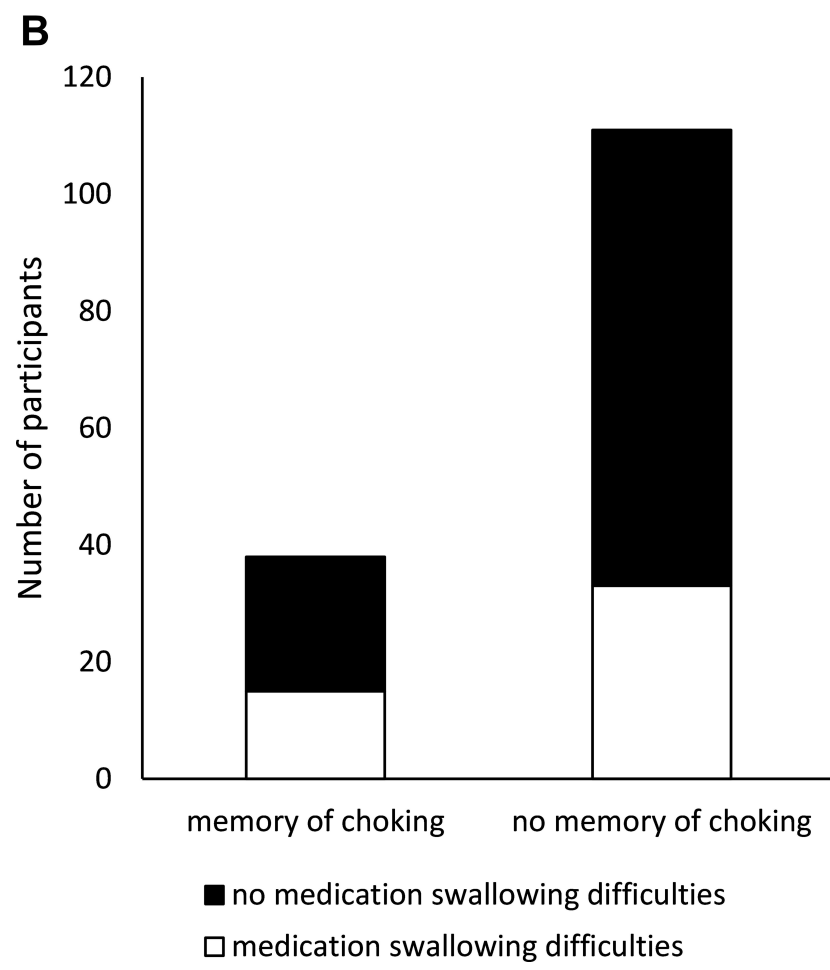

Figure I The recollection of an episode of choking on $(\mathbf{A})$ medication $(n=152)$ or (B) food for participants reporting current having medication swallowing difficulties in comparison to those with no current medication swallowing difficulties $(n=149)$. Significant difference between bars in terms of the proportion of participants reporting current medication swallowing difficulties is indicated: $*_{p}<0.05$.

mouth cavity $(46 \%, 70 / 152)$ and small mouth cavity size $(54 \%, 82 / 152)$. Most $(71 \%, 35 / 49)$ of those with current medication swallowing difficulties were classified as having a small mouth cavity while 29\% (14/49) had a large mouth cavity (Figure 2A), leading to a significant association between medication swallowing difficulties and mouth cavity size $(\mathrm{OR}=2.98, \mathrm{p}<0.05)$. Age, gender, fungiform papillae count, and the presence or absence of the gag reflex did not show any association with mouth cavity size.

The fungiform papillae density analysis was conducted on 134 participants. Four subjects did not participate in the task, and 14 were excluded due to image quality. The median number of fungiform papillae per $\mathrm{cm}^{2}$ was 13 , with a range between 0 and 41 papillae. Based on the fungiform papillae count, $16 \%(21 / 134)$ of the participants were classified as supertasters, 36\% (48/134) as medium tasters, and $48 \%(65 / 134)$ as low/non-tasters. The prevalence of current medication swallowing difficulties was significantly higher in the medium taster group and the supertaster group compared to the low/nontaster group $(\mathrm{OR}=2.57$ and 3.27, respectively, $\mathrm{p}<0.05)$ (Figure 2B). There was no correlation between the sensory factors (taste sensitivity and mouth cavity size) and the psychological factor (memory of choking).

Chewing efficiency was assessed using a confectionary jelly snake. Around half $(52 \%, 79 / 152)$ of the participants chewed their jelly snake to particle sizes greater than $4 \mathrm{~mm}$, and $32 \%(48 / 152)$ chewed to less than $4 \mathrm{~mm}$, while $16 \%(25 / 152)$ chewed to roughly equal quantities of large and small pieces (Figure 3). A greater proportion $(60 \%)$ of participants who chewed to mixed particle sizes reported having current medication swallowing difficulties than not. As such, the incidence of medication swallowing difficulties was significantly higher among those who chewed to mixed particle size compared to those who chewed to all large sizes $(60 \%$ vs $30 \%)$ or most/all small sizes $(60 \%$ vs $21 \%)(\mathrm{OR}=4.1, \mathrm{p}<0.05)$.

Only four $(3 \%)$ of the participants could not complete the swallowing task, which was to swallow one 00 sized capsule, all of whom had reported having a current problem with swallowing medications. Notably, the four participants who did not complete the capsule-swallowing task were all identified to have a small mouth cavity. One participant declined to participate in the task due to fear of vomiting if they tried to swallow the capsule. One participant made an effort to swallow the capsule but could not proceed after two further attempts due to a choking sensation. Two participants attempted to swallow but then refused because they needed food to swallow the pills. During the capsule swallow task, most participants $(83 \%$; 
Table 2 Self-Perceived Ability to Swallow Capsules of Various Sizes

\begin{tabular}{|l|l|l|l|l|}
\hline \multirow{2}{*}{$\begin{array}{l}\text { Capsule } \\
\text { Size }\end{array}$} & $\begin{array}{l}\text { Standard Closed Capsule } \\
\text { Body Length (mm) }\end{array}$ & \multicolumn{2}{|l|}{ \% of Participants } \\
\cline { 3 - 5 } & & $\begin{array}{l}\text { With Current Medication } \\
\text { Swallowing Difficulties (n=49) }\end{array}$ & $\begin{array}{l}\text { Without Current Medication } \\
\text { Swallowing Difficulties (n=1 03) }\end{array}$ & $\begin{array}{l}\text { All } \\
\text { Participants } \\
\text { (n=152) }\end{array}$ \\
\hline 4 & 14.3 & 100 & 100 & 100 \\
3 & 15.9 & 96 & 94 & 95 \\
2 & 18.0 & 94 & 93 & 93 \\
1 & 19.4 & 92 & 93 & 93 \\
0 & 21.7 & 80 & 87 & 85 \\
00 & 23.3 & 63 & 82 & 76 \\
000 & 26.1 & 51 & 69 & 63 \\
\hline
\end{tabular}

125/151) exhibited a neutral posture with their head facing forward, while 17\% (26/151) tilted their head upwards. The majority $(82 \%, 123 / 151)$ were observed to place the capsule on their tongue at the front of the mouth and very few participants placed the capsules either at the middle $(10 \%, 15 / 151)$ or at the back of the dorsum $(8 \%, 12 / 151)$. Neither head position or location of the capsule on the tongue were different between those with and without medication swallowing difficulties. Most (92\%, 136/148) of the participants took the option of using water to swallow the capsule. The cups had a nominal volume of $200 \mathrm{~mL}$ but were considered to be "full" with approximately $150 \mathrm{~mL}$, half full with approximately $90 \mathrm{~mL}$, and a quarter full with $40 \mathrm{~mL}$ of water. Most of the participants $(79 / 148 ; 53 \%)$ used less than a quarter of a cup of water $(<$ $40 \mathrm{~mL}$ ) to swallow the capsules (Figure 4). There was no significant association between difficulty swallowing capsules and the amount of water used $(p=0.56)$.

\section{Discussion}

The prevalence of current medication swallowing difficulties among 152 participants without a diagnosed dysphagia was $32 \%$ in this study. This is higher than the prevalence reported by community pharmacy customers in another Australian study $(16.5 \%),{ }^{9}$ polypharmacy patients visiting community pharmacies in Switzerland $(22.5 \%),{ }^{35}$ and a general community survey in Brazil $(17 \%) .{ }^{6}$ However, it is similar to a Danish study among adolescents $(32 \%)^{7}$ Even more of our participants (55\%) reported having found it difficult to swallow tablets or capsules at some point in their life. These findings are in line with a previous survey of general practices in Germany with $27 \%$ having current medication swallowing difficulties and $37 \%$ reporting having had medication swallowing difficulties at some point. ${ }^{11}$ This may indicate that it is possible for people to improve in their level of comfort and ability to swallow solid dosage forms.

One-third of participants $(32 \%)$ reported crushing tablets at some time to make swallowing easier. Modifying medications was more likely to occur by those reporting difficulties swallowing whole tablets. Prevalence of medication modification varies across studies and has been reported by $11-30 \%$ of general community patients. ${ }^{9,36}$ In many of these reports, swallowing difficulties was the reason for modifying medications which raises concern around the safety of medication modification by patients. 9,36,37 Modifying medications can result in dose variations, alter drug absorption, and lead to adverse events. ${ }^{38,39}$ Understanding and resolving medication swallowing difficulties can potentially prevent the risks associated with modifying dosage forms.

The findings of this study identified significant associations between certain physiological factor, namely oral cavity size and fungiform papillae density, with medication swallowing difficulties. Participants who had smaller oral cavities, in which the tongue took up more of the available space according to the modified Mallimpati assessment, were more likely to report current medication swallowing difficulties. A larger mouth cavity may support easy swallowing through less exposure to areas of high sensation in the mouth such as the tongue and papillae structures. Greater fungiform papillae density was significantly associated with self-reported medication swallowing difficulties suggesting that subjects with increased sensory awareness could be more sensitive to the taste of medications. Indeed, nearly a quarter of the general practice population report taste as the reason for medication swallowing difficulties which can lead to non-adherence. ${ }^{11}$ Overall, 14\% of our participants were classified as supertasters, which is similar to the $16 \%$ of adults in a study in 


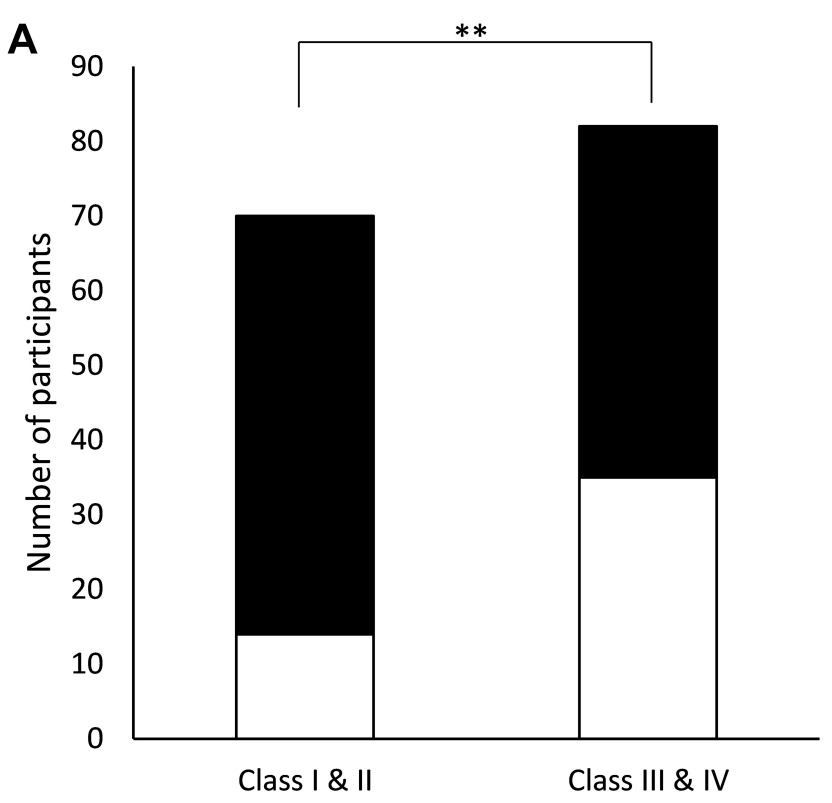

no medication swallowing difficulties $\square$ medication swallowing difficulties

\section{B}

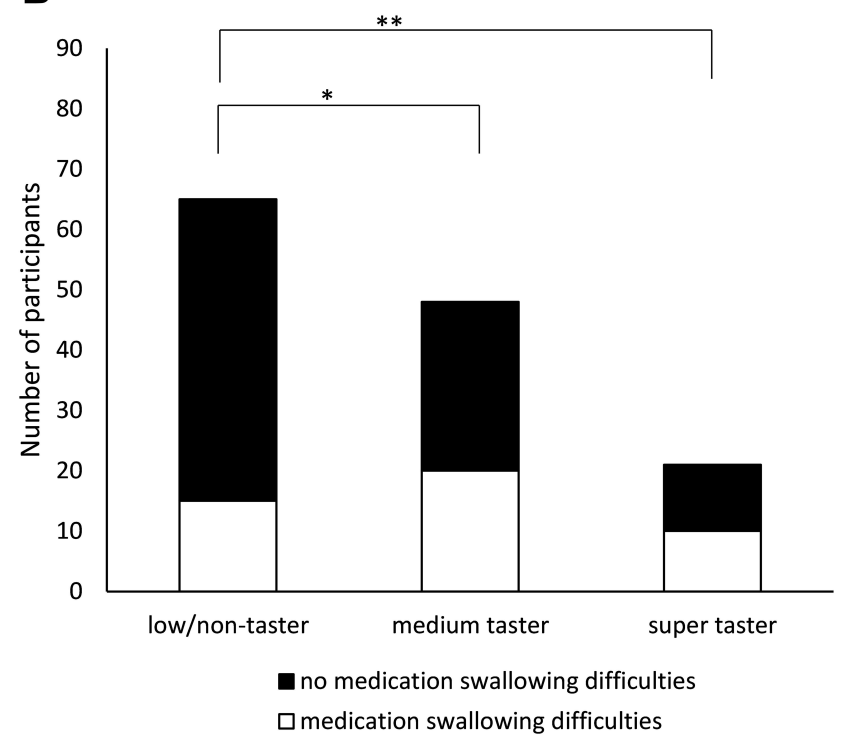

Figure 2 Mallampati classification of the oral cavity $(\mathbf{A})$ and classification of fungiform papillae density (B) for participants with or without current medication swallowing difficulties. Participants were classified as having a large mouth cavity (Class I and II) or a smaller mouth cavity (Class III and IV) according to the modified Mallampati method $(n=152)$, and as a supertaster, medium taster, and non-taster according to fungiform papillae density $(n=134)$. Significant differences between bars in terms of the proportion of participants reporting current medication swallowing difficulties are indicated: ${ }^{*} \mathrm{p}<0.01,{ }^{*} \mathrm{p}<0.05$.

southern Italy ${ }^{40}$ but slightly lower than $25 \%$ which is typical of supertasters distribution in adult populations. ${ }^{41}$ More recently developed pill-swallowing aids, such as pill-swallowing gels, sprays, or pill coatings, offer taste concealing properties that may help overcome the aversion to swallowing medications in those sensitive to the taste of medications. $^{3}$

Texture can be as important as taste in the sensory perception of food especially in swallowing difficulties ${ }^{42}$ and can be the reason for aversion to certain types of food, and surface texture of medications has been reported by more than $70 \%$ of the general practice patients as a major reason for medication swallowing difficulties. ${ }^{11}$ A positive correlation was identified for current medication swallowing difficulties with a lower preference score for potato crisps, popcorn, and granola bars, which are hard, dry, have low moisture content, and are particulate in texture. As such, medications with a rough or unpleasant surface texture may be particularly difficult for some people to swallow. ${ }^{2,10,11}$ We also considered whether people who find it difficult to swallow whole medications tend to chew their food to smaller particle sizes; however, there was no difference in reporting of medication swallowing difficulties in subjects who chewed their jelly to mostly/all small and all large size pieces. In fact, the rate of medication swallowing difficulties was significantly higher in the group who produced mixed/non-homogeneous particle sizes (large and small). This may indicate the homogeneity of the final bolus being more important than the actual particle size generated in predicting medication swallowing difficulties. It may also indicate that humans encode food textures differently to solid dose medications and that food properties cannot be used to predict tolerance of solid dose medicines.

The vast majority of the participants who thought they would not be able to swallow a size 00 capsule did in fact complete the swallowing task. This highlights the psychological barriers that many people with medication swallowing difficulties experience. People with current medication swallowing difficulties felt significantly less confident in swallowing capsule sizes of 000 and 00 as the largest size of capsules. This is in line with the findings of another study investigating the threshold size of tablets and capsules for swallowing. ${ }^{43}$ This study reported that patients found capsules/tablets larger than $21 \mathrm{~mm}$ difficult to swallow. ${ }^{43}$ Therefore, it is advisable to avoid these two capsule sizes in patients with medication swallowing difficulties. ${ }^{10,44}$ People who choose to modify medications before swallowing may be able to swallow their medications whole if they receive appropriate training, instructions, and supervision to overcome their initial fears and concerns. In this case, the presence of the speech pathologist at the swallowing task may have provided 


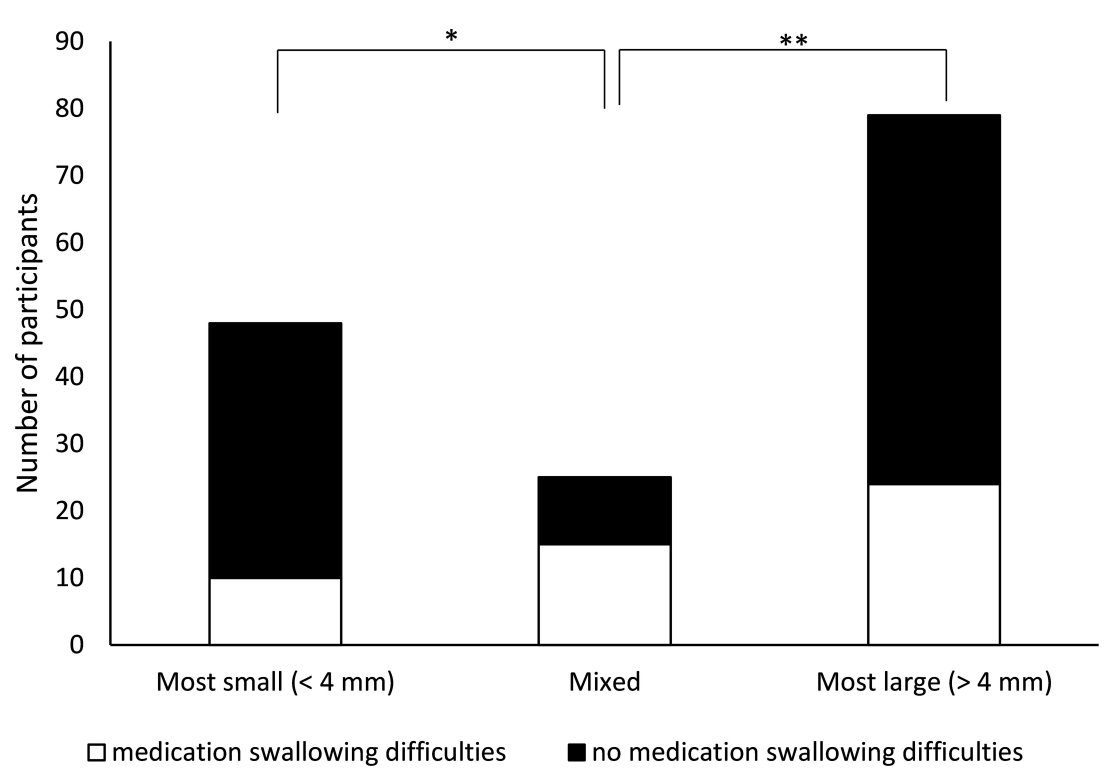

Figure 3 Particle size of bolus fragments after chewing a piece of a jelly snake $(n=152)$, for those with and without medication swallowing difficulties. Significant difference between bars in terms of proportion of participants reporting current medication swallowing difficulties are indicated: $* * p<0.01, *_{p}^{*}<0.05$.

reassurance helping them to overcome their psychological barrier to swallowing the large capsule. Training is an important and effective way to improve medication swallowing. Indeed, $18 \%$ of our participants reported having had trouble learning to swallow medications. Specific training programs for young adults and children have achieved successful and sustainable outcomes in improving medication swallowing difficulties by $75-90 \%$. ${ }^{4,46}$

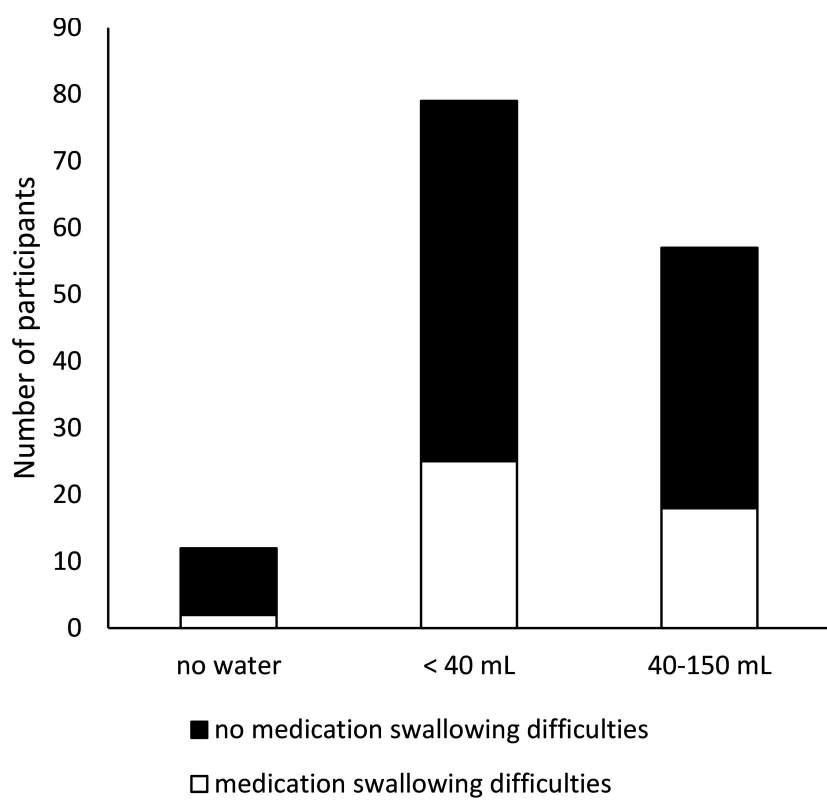

Figure 4 The quantity of water used to swallow a capsule (size 00 ) in subjects with and without medication swallowing difficulties $(n=148)$.
Having a previous episode of choking on medications was found to be a potential indicator of current medication swallowing difficulties in this study. The resulting anxiety around the potential to choke can stimulate the autonomic nervous system leading to muscle tension and inhibition of saliva flow, which thereby affects the swallowing process. $^{47}$ Anxiety or fear of swallowing medications may arise from gagging and choking on medication. ${ }^{11,37}$ Some medications, such as antibiotics, bisphosphonates, and NSAIDs can cause tissue damage when they come in contact with the esophagus ${ }^{27}$ which may also leave a negative memory leading to avoidance of swallowing medications. Having a larger proportion of people with medication swallowing difficulties with a memory of choking on medications than foods in this study indicates that swallowing of food and medications may be conceptualized differently.

The analysis of medication-taking behaviours indicated that while the majority of the participants (83\%) held appropriate head positioning during swallowing, more than half used less than the recommended amount of water for medication swallowing. The volume of liquid used for swallowing is influenced by the size of the capsule/tablets being swallowed. ${ }^{48}$ The minimum recommended volume for swallowing and safely transporting tablets and capsules through the esophagus is 50-60 mL. ${ }^{29,31}$ Quantities lower than $30 \mathrm{~mL}$ may cause the capsules to adhere to the esophageal membrane and impede the transport. ${ }^{28}$ An upright posture and neutral 
head position are also critical for safe medication swallowing. ${ }^{29,31}$ Swallowing with the head in extension can result in medication aspiration observed in patients swallowing endoscopic capsules, ${ }^{49}$ whilst the chin-down position promotes faster esophageal transit time. ${ }^{28,30}$ Although none of these factors were significantly associated with medication swallowing difficulties, patient education regarding safe and correct medication swallowing behaviours can be beneficial.

\section{Limitations}

We recognize a number of limitations in the methods used in this study. The swallowing assessments were performed by one investigator, an experienced speech pathologist, but inclusion of a second observer would have increased the validity of the results. The Mallampati classification of mouth cavity size may be influenced by obesity and ethnicity, ${ }^{17,50}$ which were not considered in this study. Moreover, the Mallampati classification is originally designed and purposed for predicting difficult tracheal intubation while there is not well-established evidence around its association with the swallowing function. In the absence of other feasible methods to classify the oral cavity size, this study utilised the Mallampati classification as an easily applied indirect indicator of mouth cavity size. Using a single product may not accurately reflect an individual's chewing efficiency for different foods, as particle size distribution is highly dependent on the type of food presented. Furthermore, the choice of $4 \mathrm{~mm}$ size limit was based on the upper limit of the median particle size for swallowing carrots in a previous study, ${ }^{33}$ which contrasts in taste, texture, and consistency with the jelly snake in our study. Participants were observed by the investigator during the chewing and capsule swallow tasks, which may have affected participant behavior. Additionally, the swallowing task involved a capsule, which is often preferred due to easier swallow than tablets, ${ }^{7}$ so the use of tablets may have produced different outcomes. Finally, the significant association between a memory of choking on medications and medication swallowing difficulties was based on the assumption that anyone that had choked on a medication in the past would have considered themselves to have had a difficulty swallowing medications at some time; this may not be the case, but the questionnaire did not require all participants to answer the question regarding having a memory of choking on medication. Future studies involving larger sample sizes are required to establish associations between medication swallowing difficulties with physiological and psychological factors.

\section{Conclusion}

The findings of this study shed light on the problems affecting medication swallowing in healthy adults without dysphagia. Medication swallowing difficulties were a common problem leading to medication modification. Having a small anatomical size of the oral cavity, greater number of fungiform papillae structures, and a memory of choking on medications were associated with current medication swallowing difficulties. People with medication swallowing difficulties felt significantly less confident to swallow larger size capsules (size 000 and 00 ), though most of them were able to swallow a size 00 capsule with water when requested to during the study. However, no significant associations were found between the presence of the gag reflex, the memory of a choking on food, or food neophobia with medication swallowing difficulties. Moreover, medication swallowing difficulties did not have a significant association with the quantity of water used for swallowing medications or the head posture when swallowing a capsule. Further research is needed to address underlying reasons for medication swallowing difficulties and inform effective strategies to overcome these issues.

\section{Acknowledgments}

The authors acknowledge the staff and students within the School of Pharmacy, University of Queensland, Australia, and staff of the Health and Food Sciences Precinct, Queensland, Australia, for participating in the study.

\section{Funding}

This research received no specific grant from any funding agency in the public, commercial or not-for-profit sectors.

\section{Disclosure}

The authors report no conflicts of interest in this work.

\section{References}

1. Stewart KD, Johnston JA, Matza LS, et al. Preference for pharmaceutical formulation and treatment process attributes. Patient Prefer Adherence. 2016;10:1385-1399. doi:10.2147/PPA.S101821

2. Liu F, Ghaffur A, Bains J, et al. Acceptability of oral solid medicines in older adults with and without dysphagia: a nested pilot validation questionnaire based observational study. Int J Pharm. 2016;512 (2):374-381. doi:10.1016/j.ijpharm.2016.03.007

3. Forough AS, Lau ET, Steadman KJ, et al. A spoonful of sugar helps the medicine go down? A review of strategies for making pills easier to swallow. Patient Prefer Adherence. 2018;12:1337-1346. doi:10.2147/PPA.S164406

4. Kappelle WF, Siersema PD, Bogte A, Vleggaar FP. Challenges in oral drug delivery in patients with esophageal dysphagia. Expert Opin Drug Deliv. 2016;13(5):645-658. doi:10.1517/17425247.2016.1142971 
5. Carnaby-Mann G, Crary M. Pill swallowing by adults with dysphagia. Arch Otolaryngol Head Neck Surg. 2005;131 (11):970-975. doi:10.1001/archotol.131.11.970

6. Souza LF, Nascimento WV, Alves LMT, et al. Medication swallowing difficulties in people without dysphagia. Rev CEFAC. 2019;21(4): e0119. doi:10.1590/1982-0216/20192140119

7. Overgaard ABA, Moller-Sonnergaard J, Christup LL, et al. Patients' evaluation of shape, size and colour of solid dosage forms. Pharm World Sci. 2001;23(5):185-188. doi:10.1023/a:1012050931018

8. Hansen DL, Tulinius D, Hansen EH. Adolescents' struggles with swallowing tablets: barriers, strategies and learning. Pharm World Sci. 2008;30:65-69. doi:10.1007/s11096-007-9142-y

9. Lau ETL, Steadman KJ, Mak M, et al. Prevalence of swallowing difficulties and medication modification in customers of community pharmacists. J Pharm Pract Res. 2015;45(1):18-23. doi:10.1002/ jppr. 1052

10. Fields J, Go JT, Schulze KS. Pill properties that cause dysphagia and treatment failure. Curr Ther Res. 2015;77:79-82. doi:10.1016/j. curtheres.2015.08.002

11. Schiele JT, Quinzler R, Klimm HD, et al. Difficulties swallowing solid oral dosage forms in a general practice population: prevalence, causes, and relationship to dosage forms. Eur J Clin Pharmacol. 2013;69(4):937-948. doi:10.1007/s00228-012-1417-0

12. Cichero JAY, Murdoch B. Dysphagia Foundation, Theory, and Practice. New York: John Wiley and Sons; 2006.

13. Davies AE, Stone SP, Kidd D, et al. Pharyngeal sensation and gag reflex in healthy subjects. Lancet. 1995;345(8948):487-488. doi:10.1016/s0140-6736(95)90584-7

14. van der Glas H, van der Bilt A, Abbink JH, et al. Functional role of oral reflexes in chewing and biting: phase-, task- and site-dependent reflex sensitivity. Arch Oral Biol. 2007;52(4):365-369. doi:10.1016/j. archoralbio.2006.10.022

15. Mishellany A, Woda A, Labas R, et al. The challenge of masticate: preparing a bolus suitable for deglutition. Dysphagia. 2006;21 (2):87-94. doi:10.1007/s00455-006-9014-y

16. Jalabert-Malbos ML, Mishellany-Dutour A, Woda A, et al. Particle size distribution in the food bolus after mastication of natural foods. Food Qual Prefer. 2007;18(5):803-812. doi:10.1016/j. foodqual.2007.01.010

17. Hashmi NK, McCartney S, Jones LH, Bartz RR. Emergency airway management. In: Brown DL, editor. Cardiac Intensive Care. Philadelphia, PA: Elsevier; 2018:525-547.

18. Frank ME, Hettinger TP, Mott AE. The sense of taste: neurobiology, aging, and medication effects. Crit Rev Oral Biol Med. 1992;3 (4):371-393. doi:10.1177/10454411920030040401

19. Pauloski BR, Nasir SM. Orosensory contributions to dysphagia: a link between perception of sweet and sour taste and pharyngeal delay time. Physiol Rep. 2016;4(11):e12752. doi:10.14814/ phy2.12752

20. Lee YM, Prescott J, Kim KO. PROP taster status and the rejection of foods with added tastants. Food Sci Biotechnol. 2008;17 (5):1066-1073

21. Lin JY, Arthurs J, Reilly S. Conditioned taste aversions: from poisons to pain to drugs of abuse. Psychon Bull Rev. 2017;24(2):335-351. doi:10.3758/s13423-016-1092-8

22. Pliner P, Hobden K. Development of a scale to measure the trait of food neophobia in humans. Appetite. 1992;19(2):105-120. doi:10.1016/0195-6663(92)90014-W

23. Van Kleef E, Van Trijp HC, Luning P, et al. Consumer-oriented functional food development: how well do functional disciplines reflect the 'voice of the consumer'? Trends Food Sci Tech. 2002;13 (3):93-101. doi:10.1016/S0924-2244(02)00068-7

24. Weaver MR, Brittin HC. Food preferences of men and women by sensory evaluation versus questionnaire. Fam Consum Sci Res J. 2001;29(3):288-301. doi:10.1177/1077727X01293006
25. Harris Interactive Inc. Pill-swallowing problems in America: a national survey of adults. New York, NY: Harris Interactive Inc. for Schwarz Pharma; 2004. 1-39. Available from: http://www.prnewswire.com/ news-releases/40-of-american-adults-report-experiencing-difficultyswallowing-pills-58973047.html. Accessed December 23, 2020

26. Macdonald J, Barker B, Hambridge J. Novel use of placebo tablets in the treatment of choking phobia. Aust J Hosp Pharm. 2001;31 (2):121-122. doi:10.1002/jppr2001312121

27. O’Neill JL, Remington TL. Drug-induced esophageal injuries and dysphagia. Ann Pharmacother. 2003;37(11):1675-1684. doi:10.1345/ aph.1D056

28. Kasashi K, Tei K, Totsuka Y, et al. The influence of size, specific gravity, and head position on the swallowing of solid preparations. Oral Sci Int. 2011;8(2):55-59. doi:10.1016/S1348-8643(11)00028-0

29. Channer K, Virjee J. Effect of posture and drink volume on the swallowing of capsules. Br Med J (Clin Res Ed). 1982;285 (6356):1702. doi:10.1136/bmj.285.6356.1702

30. Schiele JT, Schneider H, Quinzler R, et al. Two techniques to make swallowing pills easier. Ann Fam Med. 2014;12(6):550-552. doi:10.1370/afm.1693

31. Osmanoglou E, Voort I, Fach K, et al. Oesophageal transport of solid dosage forms depends on body position, swallowing volume and pharyngeal propulsion velocity. Neurogastroenterol Motil. 2004;16 (5):547-556. doi:10.1111/j.1365-2982.2004.00541.x

32. Shahbake M, Hutchinson I, Laing DG, et al. Rapid quantitative assessment of fungiform papillae density in the human tongue. Brain Res. 2005;1052(2):196-201. doi:10.1016/j.brainres.2005.06.031

33. Woda A, Nicolas E, Mishellany-Dutour A, et al. The masticatory normative indicator. J Dent Res. 2010;89(3):281-285. doi:10.1177/ 0022034509357022

34. Von Elm E, Altman DG, Egger M, et al. The strengthening the reporting of observational studies in epidemiology (STROBE) statement: guidelines for reporting observational studies. Ann Intern Med. 2007;147(8):573-577. doi:10.7326/0003-4819-147-8-20071016000010

35. Marquis J, Schneider MP, Payot V, et al. Swallowing difficulties with oral drugs among polypharmacy patients attending community pharmacies. Int J Clin Pharm. 2013;35(6):1130-1136. doi:10.1007/ s11096-013-9836-2

36. Rodenhuis N, De Smet PAGM, Barends DM. The rationale of scored tablets as a dosage form. Eur J Pharm Sci. 2004;21:305-308. doi:10.1016/j.ejps.2003.10.018

37. Mc Gillicuddy A, Kelly M, Crean AM, et al. Understanding the knowledge, attitudes and beliefs of community-dwelling older adults and their carers about the modification of oral medicines: a qualitative interview study to inform healthcare professional practice. Res Social Adm Pharm. 2019;15(12):1425-1435. doi:10.1016/j.sapharm.2019.01.004

38. Forough AS, Lau ETL, Steadman KJ, et al. Appropriateness of oral dosage form modification for aged care residents: a video-recorded observational study. Int J Clin Pharm. 2020. doi:10.1007/s11096020-01036-X

39. Thong MY, Manrique YJ, Steadman KJ, Ito E. Drug loss while crushing tablets: comparison of 24 tablet crushing devices. PLoS One. 2018;13(3):e0193683. doi:10.1371/journal.pone.0193683

40. Negri R, Di Feola M, Di Domenico S, et al. Taste perception and food choices. J Pediatr Gastroenterol Nutr. 2012;54(5):624-629. doi:10.1097/MPG.0b013e3182473308

41. Robino A, Mezzavilla M, Pirastu N, et al. A population-based approach to study the impact of PROP perception on food liking in populations along the Silk Road. PLoS One. 2014;9(3):e91716. doi:10.1371/journal.pone.0091716

42. Steele CM, Alsanei WA, Ayanikalath S, et al. The influence of food texture and liquid consistency modification on swallowing physiology and function: a systematic review. Dysphagia. 2015;30(1):2-6. doi:10.1007/s00455-014-9578-x 
43. Kabeya K, Satoh H, Hori S, et al. Threshold size of medical tablets and capsules: based on information collected by Japanese medical wholesaler. Patient Prefer Adherence. 2020;14:1251-1258. doi:10.2147/PPA.S253663

44. U.S. Department of Health and Human Services, Food and Drug Administration. Size, shape, and other physical attributes of generic tablets and capsules guidance for industry; 2015. Available from: https://www.fda.gov/media/87344/download. Accessed December 23, 2020.

45. Beck MH, Cataldo MD, Slifer KJ, et al. Teaching children with attention deficit hyperactivity disorder (ADHD) and autistic disorder (AD) how to swallow pills. Clin Pediatr. 2005;44:515. doi:10.1177/ 000992280504400608

46. Meltzer E, Welch M, Ostrom N. Pill swallowing ability and training in children 6 to 11 years of age. Clin Pediatr. 2006;45(8):725-733. doi: $10.1177 / 0009922806292786$
47. Pluess M, Conrad A, Wilhelm FH. Muscle tension in generalized anxiety disorder: a critical review of the literature. J Anxiety Disord. 2009;23(1):1-11. doi:10.1016/j.janxdis.2008.03.016

48. Fuchs J. The amount of liquid patients use to take tablets or capsules. Pharm Pract. 2009;7(3):170-174. doi:10.4321/s188636552009000300007

49. Choi HS, Kim JO, Kim HG, et al. A case of asymptomatic aspiration of a capsule endoscope with a successful resolution. Gut Liver. 2010;4(1):114-116. doi:10.5009/gnl.2010.4.1.114

50. Lee A, Fan LT, Gin T, et al. A systematic review (meta-analysis) of the accuracy of the Mallampati tests to predict the difficult airway. Anesth Analg. 2006;102(6):1867-1878. doi:10.1213/01.ane.0000 217211.12232 .55

\section{Publish your work in this journal}

Patient Preference and Adherence is an international, peer-reviewed, open access journal that focusing on the growing importance of patient preference and adherence throughout the therapeutic continuum. Patient satisfaction, acceptability, quality of life, compliance, persistence and their role in developing new therapeutic modalities and compounds to optimize clinical outcomes for existing disease states are major areas of interest for the journal. This journal has been accepted for indexing on PubMed Central. The manuscript management system is completely online and includes a very quick and fair peer-review system, which is all easy to use. Visit http:// www.dovepress.com/testimonials.php to read real quotes from published authors. 